\title{
La vegetación del humedal "Abras de mantequilla”. Composición florística. Bases para su restauración
}

\author{
Abras de Mantequilla Wetland Vegetation. Floristic Composition. Grounds for its Restauration
}

\author{
Elias Cuasquer ${ }^{1}$, Darwin Salvatierra ${ }^{1}$, Edwin Jimenez ${ }^{1},{ }^{\bullet}$ Herminio Boira $^{2}$
}

${ }^{1}$ Laboratorio de Geobotánica. Ingeniería Forestal. Facultad de Ciencias Ambientales, Universidad Técnica Estatal de Quevedo. Campus Manuel Haz Álvarez. Av. Quito Km 1.5 vía Santo Domingo de los Tsachilas. EC.120501. Quevedo, Ecuador. jcuasquer@uteq.edu.ec; dsalvatierra@uteq.edu.ec; ejimenez@uteq.edu.ec

${ }^{2}$ Investigador PROMETEO- Universidad Técnica Estatal de Quevedo. Campus Manuel Haz Álvarez. Av. Quito Km 1.5 vía Santo Domingo de los Tsachilas. EC.120501. Quevedo, Ecuador. Instituto Agroforestal Mediterráneo. Universidad Politécnica de Valencia. Prometeo SENESCYT (Ecuador). ${ }^{\circ}$ hboira@gmail.com

\section{Resumen}

$\mathrm{L}$ as principales agrupaciones vegetales del humedal de Abras de Mantequilla y su entorno se obtuvieron a partir de una matriz de 61 inventarios (Two-way Indicator Species Analysis; TWINSPAN) que evidenció la existencia de cuatro asociaciones características de biotopos más importantes, devenida de la fuerte eutrofización de las aguas (bajos valores de oxígeno disuelto (DO) ppm y altos para sólidos totales disueltos (Tsd) $\mathrm{mg} / \mathrm{L}$ ). La vegetación acuática flotante o enraizada (VAG) es de mayor extensión, con dos especies dominantes, Ludwigia helminthorrhyza y Eichornia crassipes, propias de hábitats acuáticos con un alto grado de eutrofización y tres variantes que dependen del nivel del agua de las praderas flotantes (VAPF) - compuesta por gramíneas (Panicum maximum, P. mertensii, y Paspalum repens) con largos rizomas que se entretejen para evitar la disgregación. La formación periférica, sometida a periodos de inundación recurrente (VBSI), se caracteriza por la presencia de arbustos y árboles del bosque seco (Xylosma benthamii, Hematoxylum campechianum), tolerantes a la inundación prolongada, y restos arvense asociada en los cultivos durante la época seca. El análisis de componentes principales (PCA) pone de manifiesto la fuerte alteración de origen antrópico. Los dos primeros factores con valores de 10.28 y 5.11, asumen sólo el 25.60\% de la varianza. De los índices de biodiversidad obtenidos, la vegetación palustre natural (Albidella nymphaeifolia, Sagittaria sagittifolia, S. lancifolia) es la que se destaca en las áreas con acuíferos subterráneos emergentes. La vegetación del bosque seco circundante (VBS) presenta una relativa homogeneidad florística y bajo índice de biodiversidad.

Palabras clave: Flora acuática, humedal, Abras de Mantequilla, fitosociología, biodiversidad.

Recibido: 25-noviembre-2014. Recibido en forma corregida: 28-septiembre-2015. Aceptado: 28-enero-2016.

Publicado como ARTÍCULO CIENTÍFICO en Ciencia y Tecnología 9(1): 17-30

Junio de 2016

\begin{abstract}
A bras de Mantequilla wetland and its surroundings' major A plant groups were obtained from a 61inventory matrix (Two-way Indicator Species Analysis; TWINSPAN), which evidenced the existence of four most important biotopes characteristic associations, resulting from strong water eutrophication (low levels of dissolved oxygen (DO) ppm and higher for total dissolved solids (TDS) $\mathrm{mg} / \mathrm{L}$ ). Aquatic vegetation, floating or rooted, (VAG) is the most extensive, with two dominant species, Ludwigia helminthorrhyza and Eichhornia crassipes, typical of aquatic habitats with a high degree of eutrophication and three variants depending on the water level in floating meadows (VAPF). It consists of grass (Panicum maximum, P. mertensii, and Paspalum repens) with long rhizomes that interweave to prevent disintegration, what favors birds. Peripheral formation, subjected to periods of recurrent flooding (VBSI), is characterized by the presence of dry forest shrubs and trees (Xylosma benthamii, Hematoxylum campechianum), tolerant to prolonged flooding, and weed remains associated in crops during the dry season. The principal component analysis (PCA) shows a strong anthropic alteration. The first two factors with values of 10.28 and 5.11, assume only $25.60 \%$ of the variance. Biodiversity indices obtained, stress that natural marsh vegetation (Albidella nymphaeifolia, Sagittaria sagittifolia, S. lancifolia) is the one prevailing in areas with emerging underground aquifers. Surrounding dry forest vegetation (DFV) presents a relative floristic homogeneity and low biodiversity index.
\end{abstract}

Key words: Aquatic plants, wetland, Abras de Mantequilla, phytosociology, biodiversity. 


\section{Introducción}

$\mathrm{L}^{2}$ vegetación de los bosques secos tropicales está determinada por un clima bimodal, con la incidencia de un periodo seco en donde la evapotranspiración supera a la precipitación, condicionando severamente el desarrollo vegetativo. Los valores de los índices bioclimáticos, en especial ombrotérmico general $\left(\mathrm{I}_{0}=1.58\right)$ y ombrotérmico en la época seca ( $\operatorname{Iod} 2=0.06)$, sitúan las formaciones boscosas en torno al humedal, dentro del tipo de bioma de sabana xérica semiárida con estructura de microbosque deciduo o hiemisilva (Rivas, 2008).

A diferencia de los bosques estacionalmente secos de la Región Tumbesina de Perú y Ecuador (Aguirre et al., 2006), los del entorno del humedal de Abras de Mantequilla están caracterizados por una disposición de recursos hídricos y edáficos variables en función de las explotaciones agrícolas y forestales (Bourliere, 1983; Gottsberger y SilberbauerGottsberger, 2006.) que, junto con la geomorfología, topografía y características físico-químicas de los suelos, juegan un papel determinante en la composición florística y en sus aspectos funcionales (Franco, 2002; Haridasan, 2008). El bosque climático en el área del humedal de Abras de Mantequilla (Figura 1) ha quedado reducido a una pequeña y fragmentada extensión de algo más del $5 \%$ de la superficie. La explotación agrícola y ganadera ha provocado, no solo su drástica reducción, si no la pérdida de biodiversidad. Solo en la Reserva Ecológica de Jauneche (cantón Palenque) se conserva la riqueza florística original de la región.

Otro factor que determina la fisonomía del paisaje, y en ella su composición florística, es la disponibilidad de agua a partir de la capa freática. Ello determina que, como ocurre en las sabanas del centro de Brasil (Eiten, 1972; Furley, 1996), exista una estrecha relación entre la topografía y la altitud, a pequeña escala, con la composición florística, incluso a veces dentro de pocos centenares de metros. De hecho, en la periferia o en los mismos cauces y lagunas, los acuíferos subterráneos pueden aflorar, creando pequeñas áreas de regeneración del medio y contribuyendo a mantener y albergar ciertas especies en peligro de extinción local, como ocurre en el humedal Abras de Mantequilla (AdM).

Estas observaciones son válidas cuando se comparan también los biotipos. En niveles superiores del gradiente topográfico, por lo general sobre profundos oxisoles, dominan los fanerófitos, constituyendo doseles estratificados y cerrados. A medida que disminuye la altitud, disminuyen los fanerófitos; los suelos, con la capa freática más próxima a la superficie, pueden llegar incluso a sufrir leves y periódicas inundaciones, dando paso a formaciones abiertas de nanofanerófitos acompañados de geófitos palustres propios de familias acuáticas o palustres (alismatáceas, ciperáceas, gramíneas y otras) y terófitos escandentes (lianas, bejucos) (Eiten, 1972; Furley, 1999). En una determinada área, como la del humedal AdM, las variaciones florísticas y estructurales a lo largo de los gradientes topográficos son asumidos, independientemente del bioclima, por las propiedades físicoquímicas del suelo, actuando algunos factores como filtro para el establecimiento de las especies (Ruggiero et al., 2006).

El régimen hídrico, en los humedales de pequeñas cuencas continentales o litorales, se considera el factor primordial determinante en la distribución de las especies y en la fisonomía del paisaje (Gottsberger and SilberbauerGottsberger, 2006; Child et al., 2010; Boira, 2013); la colmatación de los mismos, como fenómeno natural en las series de vegetación edafohigrófilas, es un proceso ligado en su velocidad a la topografía de las cubetas y a las oscilaciones del nivel de agua.

La vegetación macrofítica subacuática juega un papel determinante en las cadenas tróficas y por consiguiente en la biodiversidad. Son de especial interés las praderas sumergidas constituidas por elementos florísticos de las familias miriofiláceas, ceratofiláceas y nimfáceas, propias de aguas oligo-mesotróficas y en cuyo seno se desarrollan las fases larvarias y juveniles de numerosos macro invertebrados acuáticos (moluscos, crustáceos, anélidos, y otras). Sin embargo, su papel protector puede tener consecuencias negativas sobre los productores primarios y en especial el fitoplancton (Jones, 1990).

La densidad de fitoplancton, medida en cantidad de clorofila, está relacionada negativamente con la densidad de macrófitos acuáticos por el papel de filtro que estos ejercen sobre la luz; muchos de ellos obtienen la mayor parte de los nutrientes a través de los sedimentos (Cariguan y Kalff, 1980) siempre que el nivel de agua y las características morfológicas de las cubetas permitan el acceso. Otros absorben directamente los nutrientes de las columnas de agua en las que se desarrollan sus órganos radiculares constituyendo una competencia para el fitoplancton (Balls et al., 1985).

El efecto de los macrófitos flotantes, como la lenteja de agua (Lemna sp.) o el lirio de agua (Eichornia sp.) entre otros, puede tener un efecto sobre el fitoplancton si las poblaciones experimentan un fuerte crecimiento debido a la eutrofización, ya que cubren la lámina de agua e impiden el paso de la luz (Timms y Moss, 1984; Rorslett et al., 1986). Sin embargo, en determinadas condiciones de eutrofización, cuando la contaminación procede de pesticidas o excesivos superfosfatos en los abonos pueden tener efectos positivos, contribuyendo a la liberación de minerales en especial fósforo (Engel, 1988).

El área del humedal AdM, con aproximadamente 63.300 ha de extensión y relacionada con seis cuencas fluviales del Ecuador, alberga formaciones vegetales muy diversas, desde las propias comunidades hidrófitas y helófitas en zonas de inundación con niveles variables y estacionales, hasta restos del bosque climácico deciduo (Bosque siempreverde de tierras bajas de la Costa) MAE (2012). La categoría de "Sitio RAMSAR con el No. 1023" otorgada en el año 2000, se justifica de forma oficial por su extensión, por su función 
como sede de anidación y paso de aves nativas y migratorias, entre ellas cuatro amenazadas, y por la rica y diversa ictiofauna integrada en las cadenas tróficas. Pero además, contribuye al mantenimiento y desarrollo de los núcleos urbanos de su entorno.

En la actualidad la mayor parte de la superficie (95\%) es objeto de explotaciones agropecuarias con un fuerte impacto negativo sobre el humedal a través de la escorrentía y lixiviación de los compuestos orgánicos, fertilizantes y pesticidas efluentes de los cultivo de maíz y arroz en particular. Los estudios sobre el medio natural del humedal se han centrado fundamentalmente sobre la fauna y el fitoplancton, estos últimos a raíz de la progresiva contaminación acuática. Las referencias a la flora y a la vegetación son por lo general aisladas y forman parte de trabajos no específicos del ecosistema. Solo el bosque seco del entorno ha sido abordado en un estudio particular sobre la flora de Jauneche (Dodson et al., 2005); en él se registran más de 728 especies de plantas vasculares, en especial especies emergentes, de dosel y lianas, pero con escaso rigor en la caracterización taxonómica y diversidad de los elementos acuáticos y palustres.

En el límite inferior de estos bosques, junto a lagunas, ríos y esteros, se establecen orlas de vegetación aclaradas y sometidas al régimen de inundaciones (Rossatto et al., 2012). La cobertura de los bosques remanentes representa solo el 5\% de la superficie, consecuencia de la colonización agrícola; los parches que se encuentran están ubicados en los recintos de Campo Verde, El Abanico, El Recuerdo y San Juan de Abajo. Entre la flora más representativa se encuentra especies de los géneros de Albizia, Centrolobium, Ceiba, Triplaris, Vitex, Jatropa, Muntigia, Cordia, Manguifera, Ficus, Causopoa, Acacia y entre otros, constituyendo la flora más importante de la región conjuntamente con la Reserva Ecológica Jauneche.

La singularidad del ecosistema de Abras de Mantequilla está basado en su geomorfología que se compone por un mosaico de lomas (de 6 a 12 m de desnivel) y valles surcados por ríos de amplios canales de aguas lenticas en esteros, la inundación temporal correspondiente a la precipitación estacional y como consecuencia de ello, una alternancia en los niveles de inundación que alcanzan diferencias de hasta 5 $\mathrm{m}$. No se han llevado a cabo estudios sobre el valor ecológico de las especies vegetales, su función colonizadora así como su papel bioindicador, característico de las distintas comunidades vegetales. Mientras en los ecosistemas fluviales y lacustres de planicies y llanuras se establecen, a nivel mundial, orlas de vegetación periférica palustre, responsables de una lenta y progresiva colmatación, en el humedal de Abras, la orla la constituye una franja de bosque con especies características y adaptadas a un largo periodo de inundación. Una alteración en el régimen hídrico, como la disminución de caudales o menores intervalos de cota de inundación, favorecería el establecimiento del bosque secundario.

Considerando la ausencia de estudios específicos la vegetación y el paisaje del ecosistema del humedal Abras de Mantequilla, los objetivos propuestos en el trabajo contemplan el Estudio florístico: 1) Inventariar y analizar las principales comunidades y asociaciones de la vegetación acuática, palustre y bosque periférico inundable con sus especies características; 2) Caracterizar ecológicamente las comunidades y asociaciones vegetales a partir de parámetros fisicoquímicos de las aguas y régimen de inundación y 3 ) Determinar de la riqueza y pureza florística mediante la evaluación de la biodiversidad.

\section{Materiales y métodos}

【 a zona de estudio, representativa del actual ecosistema del humedal (Figura 1), comprende ríos, esteros y áreas periféricas a lo largo del río Vínces (Cuenca del rio Guayas) en la provincia de Los Ríos (Ecuador). Está delimitada por las coordenadas UTM: 652754E, 9655912N, en el extremo NE (Las Garzas); 646645E, $9828435 \mathrm{~N}$, en el S (Vínces) y 641081E, 9840793N, en el W (Palenque).

Los estudios se realizaron durante la época estival (época seca, septiembre a octubre, 2014) y principios de invierno (época húmeda, enero a marzo, 2015). La determinación de las muestras de plantas recolectadas, prensado y secado en el herbario de la UTEQ y para la identificación se utilizó las claves generales (Harling et al., 1973-2005) y estudios específicos (Brown, 2008). Los especímenes de interés corológico o ecológico fueron depositados en el Herbario de la UTEQ. En los inventarios de vegetación acuática se incluyeron datos fisicoquímicos correspondientes de las aguas: $\mathrm{pH}$, Ta, salinidad, oxígeno disuelto (DO), conductividad eléctrica (EC) y total sólidos disueltos (Tsd), mediante sonda multiparamétrica (HANNA mód. HI9828), como indicadores de la calidad de las mismas y su relación con la vegetación. Los inventarios fueron levantados de acuerdo al método Sigmatístico (Braun-Blanquet, 1979), incluyendo todas las especies y su valor de abundancia-dominancia. Para ello, y partiendo de una determinada superficie, según el tipo de formación vegetal que la cubre, se levantaron inventarios en los que figuran las especies y los valores de abundancia dominancia según el porcentaje de cobertura (proyección de las partes aéreas, copas en caso de árboles, sobre el suelo). Los valores de cobertura del área asignados para cada especie con un valor de: 5 para mayor del 75\%; 4, entre el 50 y $75 \%$; 3 , entre 25 y $50 \%$; 2, entre 5 y $25 \%$; “+”, individuos raros o únicos con pequeña cobertura (inferior al 1\%). En los tratamientos numéricos al signo "+" se le atribuye un valor de 0.20 .

Con la matriz de datos (especies $\mathrm{x}$ inventarios) se determinó, previa eliminación de los taxones presentes en menos del 5\% de los inventarios, los principales grupos de vegetación y sus especies características aplicando el software específico Two-Way Indicator Species Analysis (TWINSPAN: 


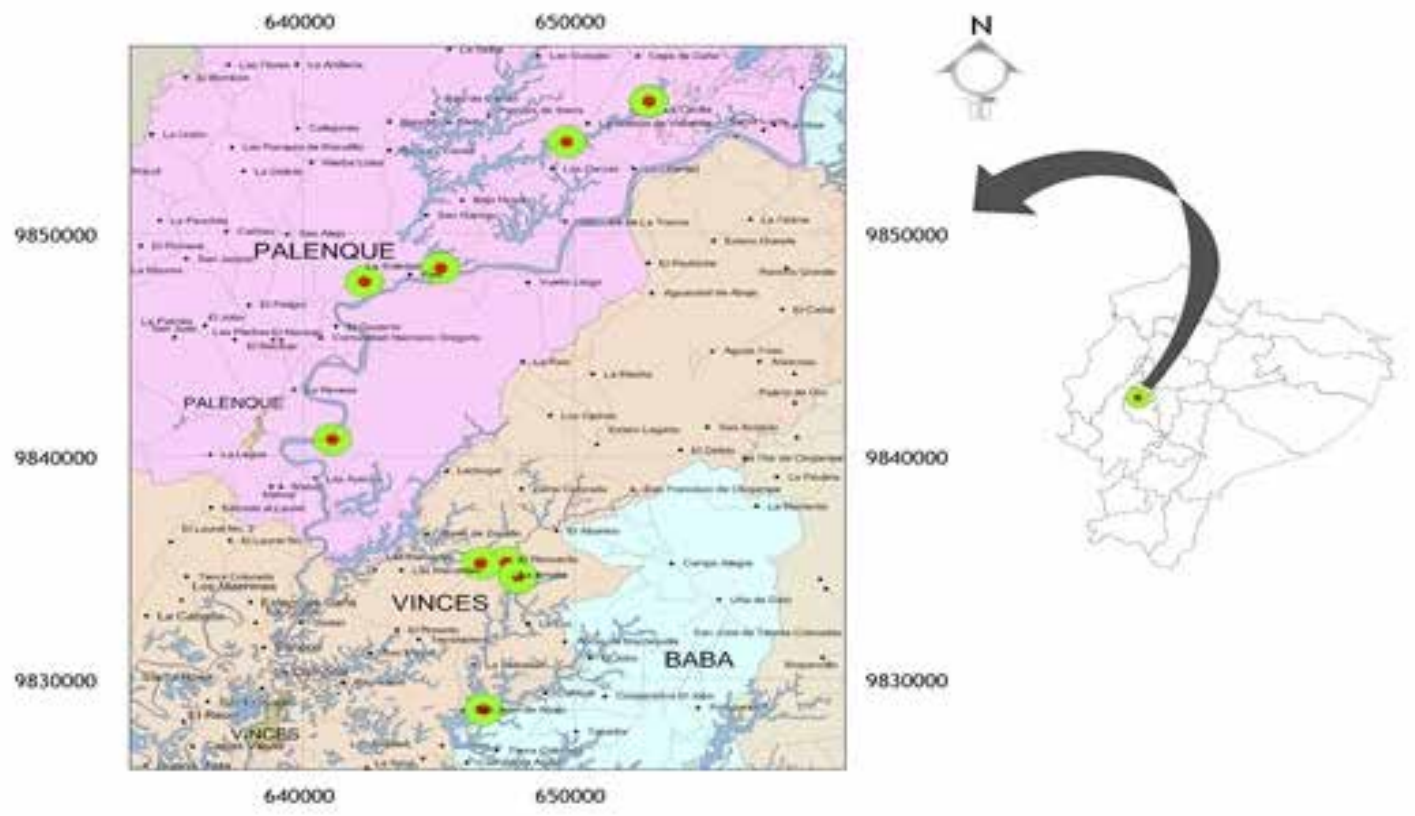

Figura 1. Zona de estudio (Los Ríos, cantones de Palenque y Vínces) y áreas de muestreo (círculos) en el humedal Abras de Mantequilla

Hill, 1979), lo que permitió crear unidades de vegetación mediante la agrupación de inventarios según las distancias florísticas y obtener valores propios significativos para las especies características de cada unidad.

Los parámetros físico-químico de las aguas que se obtuvieron en función de la presencia de hidrófitos, entre 20 y $80 \mathrm{~cm}$ de profundidad. En caso de aguas contaminadas, sin presencia de especies, los datos se registraron en función de la profundidad de la cubeta, variando entre 10 y 100 $\mathrm{cm}$. Los muestreos estuvieron ligados a los 61 inventarios correspondientes.

La relación de los factores ecológicos con las asociaciones y su estructura se obtuvieron mediante el método de componentes principales (PCA) dentro del paquete estadístico MVSP (Multivariated Statistical Pckage) (Kovacs Compueter, 2012). El mismo programa se empleó para los cálculos de biodiversidad basados en la fórmula propuesta por Shannon y Weaver (1971).

\section{Resultados y discusión}

$\mathrm{E}_{\mathrm{per}}^{\mathrm{x}}$ xceptuando la vegetación del bosque seco, no afectada por el régimen hídrico del humedal, y las especies acuáticas dominantes, bioindicadoras de un amplio grado de eutrofización (Anexo 1), las agrupaciones no se definieron en unidades discretas por la numerosa presencia de taxones de amplio espectro ecológico como las arvenses en cultivos periféricos y las acuáticas invasoras cosmopolitas.

Los resultados del análisis de grupos (TWINSPAN) permitieron diferenciar cinco comunidades vegetales y sus correspondientes variantes. Tres asociaciones forman parte de la serie de vegetación edafohigrófila; una de ellas, (1) vegetación palustre primaria (VPP), relíctica y presente en hábitats fragmentados, contiene especies de alto valor corológico, encontrándose en pequeñas charcas originadas por el afloramiento de acuíferos o manantiales a veces en el interior de parcelas de cultivo; una segunda, (2) vegetación acuática praderas flotantes (VAPF), es de carácter estable y flotante; asume la función de residencia o nicho de aves, fundamentalmente. (3) Las formaciones acuáticas en general (VAG) son de mayor extensión en el humedal, reflejan por el contrario el estado de degradación del mismo, ya que en ellas predominan especies invasoras con una fuerte capacidad de expansión y colonización. Las dos últimas asociaciones obtenidas corresponden al (4) bosque seco (VBS) y a una franja inferior del mismo sometida a las inundaciones periódicas y constituyen la orla exterior en áreas no alteradas por prácticas agrícolas (VBSI) (5).

Los factores que las definen son numerosos y con valores propios bajos. De los resultados obtenidos del PCA, basados en los coeficientes de correlación de los inventarios, los dos primeros factores se observaron tan solo el 25\% de la varianza. El primer factor ( $\mathrm{F} 1=17 \%$ de la varianza) separa los grupos de vegetación acuática de los típicos del bosque seco, mientras que el segundo $(\mathrm{F} 2=8.50 \%)$ explica una gradación 


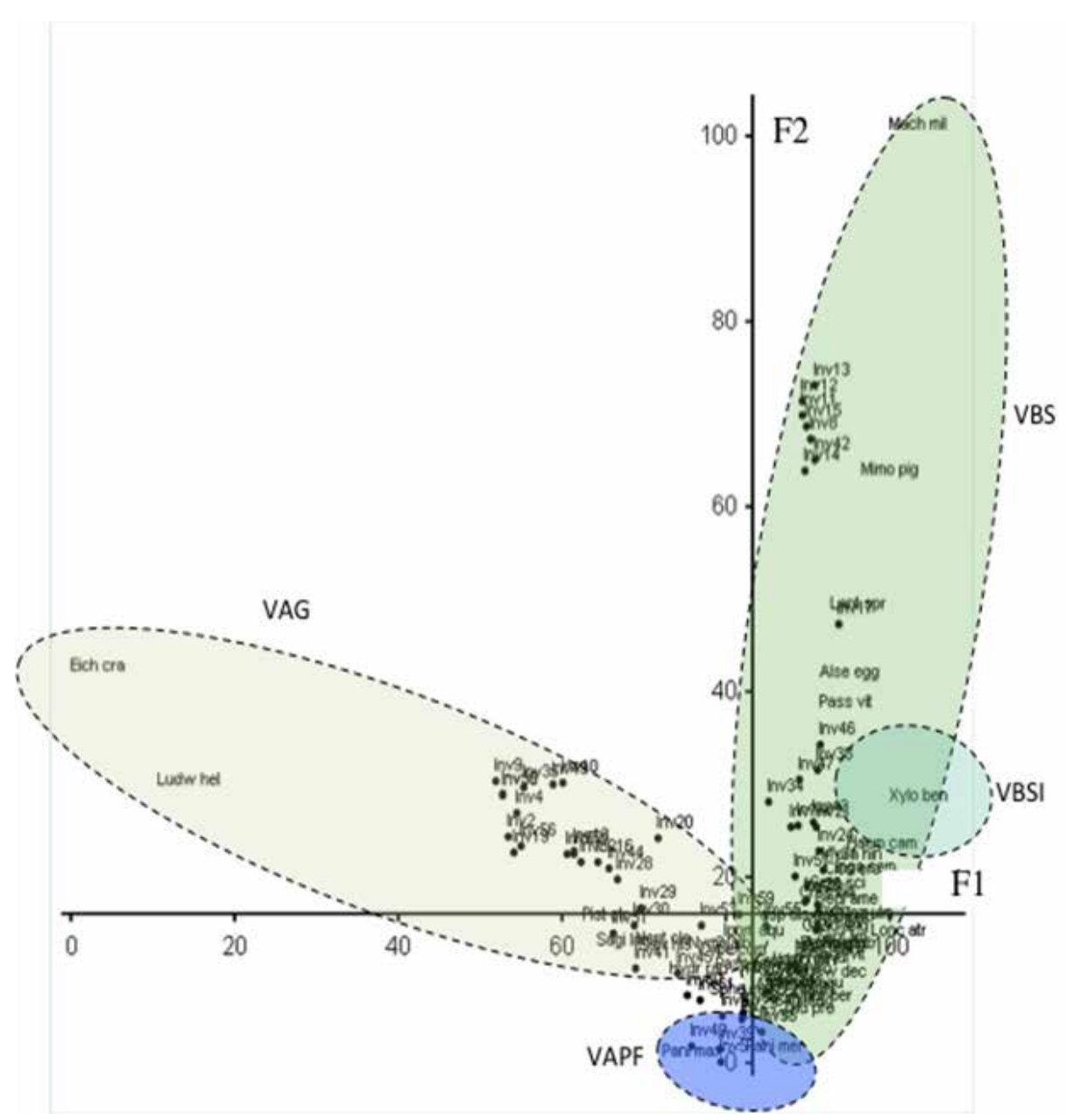

Figura 2. Grupos de vegetación distribuidos a lo largo de los dos primeros ejes extraídos del PCA(Varianza representada $F 1=17 \% ; F 2=8.50 \%)$. La comunidad VAG está ligada fundamentalmente a los valores negativos de $F 1$; en ella destacan plantas propias de aguas eutrofizadas como E. crassipes L. helminthorrhiza. En la comunidad VBS se integran especies de bosque seco relacionadas con valores positivos del eje F2 (M. Pigra (Mimo pig), L. sprucei (Lant spr) como bioindicadoras. El bosque seco inundable (VBSI) con X. benthamii (Xylo ben) y H. campechanum (Hem cam), como características del bosque seco. La vegetación de praderas flotantes (VAPF) guarda una escasa relación con los valores negativos de F2, así como la vegetación acuática primaria (VAPP), casi imperceptible por la escasa relación con ambos factores.

de comunidades acuáticas y palustres en función de variables ambientales diversas (Figura 2).

\section{Vegetación acuática errante (VAG)}

En la época seca (diciembre a enero), es la formación de mayor extensión en el humedal. Llega a ocultar prácticamente la superficie del agua en los esteros y lagunas, dificultando la navegación y creando un medio afótico que impide la productividad primaria, en especial del plancton (Romo y Miracle, 1994; Nõges et al., 2003).

La fuerte eutrofización y las variaciones superficiales de la lámina de agua, favorecen los mecanismos de crecimiento y reproducción de las especies neocolonizadoras, comunes en casi todas las zonas estudiadas, (Eichornia crassipes,
Ludwigia helminthorrhiza) y en menor frecuencia, pero con los mismos rasgos ecológicos, sus acompañantes (Salvinia hispida y Neptunia oleraceae; Cuadro1, Inv. 1-12).

Desde las zonas centrales de los esteros hasta las orillas se incorporan a las especies mencionadas distintos taxones auspiciados por rasgos de las cubetas, como la profundidad y la pendiente entre otros, y que permiten diferenciar tres subasociaciones. Nymphaea glandulifera se presenta como diferencial de las zonas interiores más profundas (1-2 m; Cuadro 1, Inv. 13 a 16) y por lo general con menos eutrofización. En estas circunstancias es baja la riqueza florística. A medida que disminuye la profundidad y en las inmediaciones de las orillas, se instalan grandes poblaciones flotantes de lirios de agua (Eichornia sp., Pistia stratioides, Sagittaria sp, Neptunia 
aquatica, y otros), siendo la especie Sagittaria lancifolia la diferencial del subgrupo (Cuadro 1, Inv. 18 a 22).

A medida que disminuye el nivel del agua y se entra en zona de contacto con los cultivos, aparece como dominante y característica de este subgrupo Paspalum maximum (Cuadro 1, Inv. 23 a 28), acompañada de un cortejo variable de especies palustres (Ipomoea aquatica, Ipomoea carnea) o arvenses y ruderales (Oenothera villosa, Cyperus compactus, Sphenoplea ceylanica, y otros).

Este tipo de vegetación es probablemente el que mejor refleja la elevada degradación del medio. En el análisis de componentes principales (PCA), los valores propios de los factores extraídos son muy bajos; la suma de los primeros 5 factores alcanza solo a explicar el $46.60 \%$ de la variabilidad total. La presencia de taxones acuáticos o palustres de amplio espectro ecológico pero favorecidos por la elevada concentración de nutrientes, regímenes de inundación variable, pesticidas etc., crea una distribución diluida a lo largo del eje principal (Figura 1; Grupo VAG).

La presencia constante y alta densidad de Panicum maximum, a pesar de ser un bioindicador de estado regresivo (Soriano, 2010), ejerce un papel positivo sobre la recuperación de la macro fauna palustre en lechos degradados por aguas efluentes de actividades humanas (Pétémanagnan et al., 2011). Esta formación es favorecida por la alta concentración de nitratos y fosfatos en el agua. Las causas de su dominancia hay que atribuirlas a las transformaciones agrícolas y ganaderas principalmente y a su constante vertido de nutrientes y pesticidas. De algunos datos referentes a la calidad de agua (DO y TSD), tomados durante el periodo seco de 2014 (Sep. a Nov., 2014) y principios del periodo húmedo (Ene. a Feb., 2015) (Cuadro 2) se puede comprobar la baja calidad biológica de las aguas y su degradación referidos a estudios realizados previamente (Alvarez y Mynett, 2010) sobre el estado del fito y zooplancton y su relación con la eutrofización del medio. Esta circunstancia es destacable en los esteros del área de Las Garzas y Pise (Cuadro 2).

\section{Las praderas acuáticas flotantes (VAPF)}

Constituyen una unidad fisionómica y funcional que viene a sustituir a los carrizales (Phragmites sp., Scirpus sp, magnocárices, y otros) y tatorales (Typha sp, Schoenoplectus sp., Cyperus sp.) de otros humedales (Moreno-Casasola et al., 2010; Boira, 2013) en los que las oscilaciones del nivel de agua son menores y con periodicidad no tan acusada. Están formadas por especies de gramíneas con gruesos y largos rizomas que se entretejen para dar consistencia física y resistencia mecánica a la disgregación. Ello hace que, a diferencia de las formaciones de Eichornia crassipes, que se fragmentan al aumentar el nivel de agua, la superficie y la corriente, en el caso de estas praderas puedan permanecer como grandes unidades de lento movimiento y ancladas en muchos casos a las orillas.

Desde el punto de vista ecológico juegan un papel determinante como puntos de parada, descanso y anidación de numerosas aves e incluso como fuente de alimentación (Pétémanagnan et al., 2011).

La composición florística (Cuadro 3) registra, como dominantes y características, a las especies Panicum martensii y Paspalum repens, a las que se unen en determinados ecotópos, Panicum maximum y otras propias del humedal.

La plasticidad de los rizomas y su tasa de crecimiento les permite responder de forma rápida a las crecidas de caudal. La proximidad de estas praderas flotantes a las orillas de los esteros facilita la incorporación de elementos de biología similar aunque de mayor espectro ecológico (Cuadro 3; Inv. 3 y 4). Esta combinación florística esta poco relacionada con los dos primeros factores extraídos (Figura 1).

\section{La vegetación acuatica primaria (VAPP)}

Este tipo de vegetación se localiza, de forma relíctica, en determinadas áreas periféricas al humedal donde existen surgencias de acuíferos. Los inventarios levantados (Cuadro 4, Inv. 1, 2 y 3) corresponden a charcas o pequeñas balsas de agua de 5 a $10 \mathrm{~m}^{2}$ originadas por el afloramiento de agua en el interior de parcelas con cultivo de arroz. Poseen una composición florística típica, algunas de ellas poco o no reportadas para la flora del humedal. Son características: Albidella nymphaefolia, Cyperus compactus, Sagittaria lancifolia y Eleocharis elegans (Cuadro 4). El cortejo florístico está compuesto por algunas especies arvenses del cultivo del propio arroz (Pennisetum purpureum, Ambrosia peruviana, Echinochloa crus-galli, y otros) o procedentes de las formaciones acuáticas contiguas (Eichornia azurea, Pistia stratioides, Sagittaria sp., y Neptunia aquática) entre otras.

\section{El bosque seco y su orla inferior sometida a la inundación periódica (VBSI)}

Los restos de bosque seco que de forma fragmentada aparecen en el entorno inmediato del humedal presentan un estrato arbóreo mixto, más o menos aclarado, de especies caducifolias y xerófitas perennifolias como Cochlospermum vitifolium, Machaerium millei, Guazuma ulmifolia, Prosopis juliflora como frecuentes (Cuadro 5: asociación 5A). El estrato arbustivo es pobre en cobertura, con relativa frecuencia de las especies Lantana sprucei y Alseis eggersii y una presencia generalizada de especies escandentes o lianoides como Passiflora sp. Luffaoperculata, Ipomoea sp., entre otras. Los inventarios incluyen frecuentemente especies arvenses y ruderales incorporadas por la actividad ganadera o agrícola inmediata.

En determinados sectores (San Juan de Abajo, El Recuerdo, Las Garzas) y por causas diversas (topografía, tipo de explotación, y otros), el bosque seco entra en contacto con las orillas del humedal presentando una franja perimetral al mismo con un cortejo florístico singular. Las especies que colonizan, de manera destacada, este biotopo son especies de escasa presencia en el bosque típico, adaptándose a una 


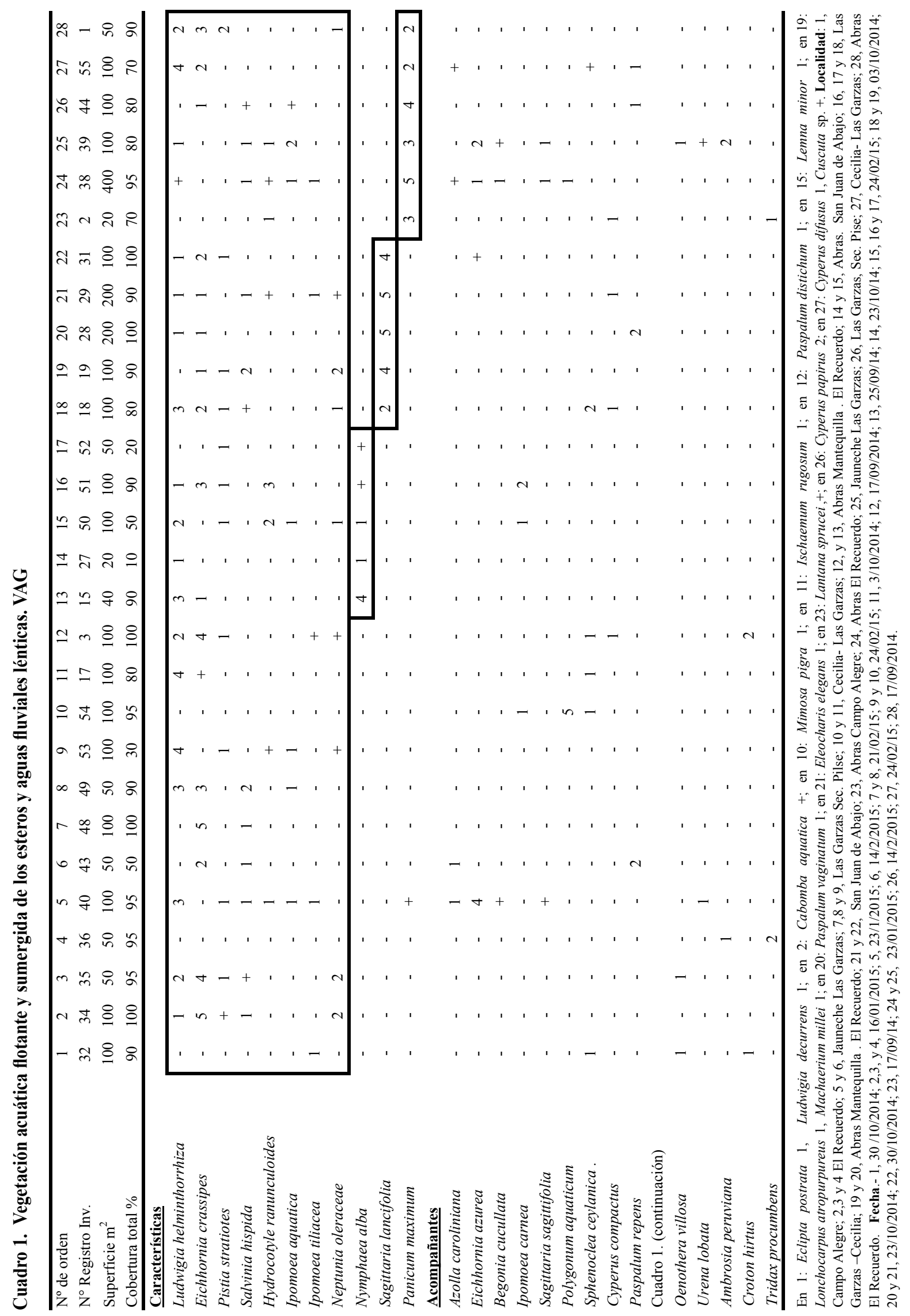


Cuadro 2. Valores significativos de los parámetros físico- químicos analizados. Oxígeno disuelto (DO) y sólidos totales disueltos (Tsd) en aguas del humedal, verano 2014-2015. Referencia a datos de 2009 (Arias et al., 2010)

\begin{tabular}{|c|c|c|c|c|c|c|c|}
\hline \multirow{2}{*}{ Loc./época } & \multicolumn{4}{|c|}{ OD ppm } & \multicolumn{3}{|c|}{ Tsd $\mathrm{mg} \mathrm{L}^{-1}$} \\
\hline & $\mathrm{n}$ & media & $(*)$ & Err. Est.. & media & $(*)$ & Err. Est. \\
\hline Datos 2009 & 12 & 6.19 & $\mathrm{a}$ & 0.81 & 105.00 & $\mathrm{~b}$ & 9.49 \\
\hline Recuerdo $14^{(1)}$ & 7 & 5.50 & $a b$ & 0.60 & 141.14 & $\mathrm{a}$ & 1.01 \\
\hline Recuerdo, Garzas y C. Alegre $15^{(2)}$ & 14 & 3.93 & $\mathrm{~b}$ & 0.89 & 73.29 & $\mathrm{~b}$ & 7.52 \\
\hline Cecilia, Pilse $15^{(3)}$ & 10 & 0.29 & $\mathrm{c}$ & 0.08 & 65.60 & $\mathrm{c}$ & 0.50 \\
\hline
\end{tabular}

(*) Medias seguidas por la misma letra no presentan diferencias estadísticas $(\mathrm{p}<0.05)$

(1) Correspondientes a los inventarios 12,13, 19, 20, 28 (Cuadro 1)

(2) Correspondientes a los inventarios 2, 3, 4, 5, 15, 16, 17, 23, 24, 25 (Cuadro 1)

(3) Correspondientes a los inventarios 7, 8, 9, 10,26, 27 (Cuadro 1)

Cuadro 3. Vegetación de praderas flotantes (hidrogeófitos) (VAPF)

\begin{tabular}{|c|c|c|c|c|}
\hline $\mathrm{N}^{\circ}$ de orden & 1 & 2 & 3 & 4 \\
\hline $\mathrm{N}^{\circ}$ Registro Inv. & 57 & 58 & 59 & 60 \\
\hline Superficie $\mathrm{m}^{2}$ & 100 & 100 & 200 & 100 \\
\hline Cobertura total \% & 100 & 100 & 100 & 100 \\
\hline \multicolumn{5}{|l|}{ Características } \\
\hline Paspalum repens & 1 & 2 & 1 & 2 \\
\hline Panicum mertensii & 5 & + & 4 & 5 \\
\hline \multicolumn{5}{|l|}{ Acompañantes } \\
\hline Azolla caroliniana & - & - & 1 & + \\
\hline Begonia cucullata & - & - & 1 & 1 \\
\hline Eichhornia crassipes & - & - & 1 & 1 \\
\hline Hydrocotyle ranunculoides & - & - & + & 1 \\
\hline Ipomoea aquatica & - & - & 1 & 1 \\
\hline Ipomoea carnea & 1 & - & - & + \\
\hline Neptunia oleraceae & - & - & 1 & 1 \\
\hline Pistia stratiotes & - & - & 1 & + \\
\hline Polygonum aquaticum & 1 & + & - & - \\
\hline Typha dominguensis & 1 & - & 1 & - \\
\hline \multicolumn{5}{|c|}{$\begin{array}{l}\text { En 1, Ludwigia helminthorrhiza +, Machaerium } \\
\text { isadelphum } 1 \text {; en 2, Mimosa pigra } 1 \text {, Panicum maximum } \\
\text { 2; en 3, Lemna minor +; en 4, Salvinia hispida +. } \\
\text { Localidad: } 1 \text { y 2, Abras Las Garzas-Cecilia; 3, Abras } \\
\text { Las Garzas-Pilse; } 4 \text { Abras Las Garzas. Fecha: } 1 \text { a 4, } \\
24 / 02 / 2015\end{array}$} \\
\hline
\end{tabular}


Cuadro 4. Vegetación acuática y palustre primaria. VAPP

\begin{tabular}{lcccc}
\hline $\mathrm{N}^{0}$ de orden & 1 & 2 & 3 & 4 \\
$\mathrm{~N}^{\circ}$ Registro Inv. & 4 & 8 & 9 & 22 \\
Superficie $\mathrm{m}^{2}$ & 2 & 8 & 20 & 10 \\
Cobertura total $\%$ & 100 & 100 & 90 & 90 \\
\hline
\end{tabular}

Características

Albidella nymphaeifolia

Cyperus compactus

Hydrocotyle ranunculoides

Eichhornia azurea

Sagittaria sagittifolia

Sagittaria lancifolia

Eleocharis elegans

$\begin{array}{cccc}1 & + & 1 & 3 \\ 1 & 1 & 1 & - \\ - & + & 1 & - \\ - & - & - & 1 \\ - & - & - & 2 \\ 2 & - & - & - \\ 1 & - & + & 1\end{array}$

Especies acuáticas invasoras, propias de medios eutrofizados

Neptunia oleraceae

Salvinia hispida

Eichhornia crassipes

Ludwigia helminthorrhiza

$\begin{array}{llll}1 & + & - & 1 \\ 1 & 1 & - & 1 \\ - & 4 & 2 & - \\ - & 2 & 1 & -\end{array}$

Especies arvenses de cultivos en arroz y maíz

Oenothera villosa

Pennisetum purpureum

Ambrosia peruviana

Cyperus aggregatus

Echinochloa crus-galli

Eclipta postrata

Ipomoea tiliacea

Matricaria chamomilla

Higrófitas de amplio espectro ecológico

Mimosa pigra

Paspalum repens

Paspalum distichum

Pistia stratiotes

Solanum flavescens

Sphenoclea ceylanica

Tecoma bernoullii

Tridax procumbens

$\begin{array}{llll}- & + & - & + \\ 2 & - & 1 & - \\ 2 & - & - & - \\ - & - & - & 1 \\ 1 & - & - & - \\ 1 & - & - & - \\ 1 & - & - & - \\ - & - & - & +\end{array}$

Localidad: 1, 2 y 3, Abras El Recuerdo; 4, Abras San Juan de Abajo. Fecha: 1, 17/09/14; 2 y 3, 25/09/14; 4, 3/10/2014. Medio- Inv. 1: acuífero junto a maíz; Invs. 2 y 3 , acuíferos en cultivo arroz; Inv. 4, acuífero junto a camino estero época seca. 
Cuadro. 5. Tablas fitosociológicas del Bosque Tropical Seco inferior en el humedal de Abras: VBS, restos de formaciones en laderas y cumbres. VBSI, franja de bosque periférica a los esteros y ríos sometida a régimen de inundación

\begin{tabular}{|c|c|c|c|c|c|c|c|c|c|c|c|c|c|c|c|c|c|c|}
\hline \multirow[b]{2}{*}{$\mathrm{N}^{\circ}$ orden } & \multicolumn{12}{|c|}{ B. seco (VBS) } & \multicolumn{6}{|c|}{ B. seco inundable (VBSI) } \\
\hline & 1 & 2 & 3 & 4 & 5 & 6 & 7 & 8 & 9 & 10 & 11 & 12 & 1 & 2 & 3 & 4 & 5 & 6 \\
\hline $\mathrm{N}^{\circ}$ registro & 6 & 7 & 8 & 11 & 12 & 13 & 14 & 21 & 48 & 42 & 26 & 25 & 17 & 22 & 38 & 43 & 46 & 47 \\
\hline Superficie $\mathrm{m}^{2}$ & 400 & 400 & 400 & 100 & 100 & 100 & 100 & 400 & 100 & 200 & 200 & 400 & 100 & 400 & 200 & 200 & 100 & 200 \\
\hline Orientación & - & $\mathrm{E}$ & $\mathrm{E}$ & $\mathrm{E}$ & NE & $\mathrm{NE}$ & $\mathrm{N}$ & $\mathrm{NE}$ & - & $\mathrm{W}$ & NW & NW & NE & $\mathrm{N}$ & - & $\mathrm{W}$ & - & - \\
\hline Pendiente \% & - & 10 & 5 & 5 & 5 & 5 & 10 & 2 & - & 15 & 5 & 5 & 10 & 10 & - & 15 & - & - \\
\hline Cobertura total $\%$ & 100 & 80 & 90 & 100 & 100 & 80 & 100 & 80 & 80 & 80 & 95 & 90 & 100 & 50 & 90 & 90 & 100 & 95 \\
\hline \multicolumn{19}{|l|}{ Especies comunes } \\
\hline Mimosa pigra & - & - & 1 & - & 1 & 2 & 5 & - & 2 & 1 & 1 & 1 & 1 & - & 2 & 1 & 2 & 2 \\
\hline Passiflora vitifolia & - & - & - & 2 & 1 & 2 & - & - & - & 1 & - & - & 1 & - & - & + & - & - \\
\hline Guadua angustifolia & 1 & - & - & - & - & - & - & 1 & - & + & - & - & 1 & + & - & - & - & - \\
\hline Gustavia pubescens & - & - & - & - & - & - & - & 1 & 1 & - & - & - & - & - & 1 & - & 1 & - \\
\hline Inga semialata & - & - & - & - & - & - & - & 2 & - & - & - & - & 2 & - & 3 & 1 & - & - \\
\hline Lonchocarpus atropurpureus & 4 & - & - & - & - & - & - & - & - & - & 5 & 5 & - & 1 & - & - & - & - \\
\hline Paspalum repens & - & - & - & - & - & - & - & - & - & - & 1 & 1 & - & - & 1 & 1 & - & - \\
\hline \multicolumn{19}{|l|}{ Características BS } \\
\hline Machaerium millei & - & 1 & 2 & 5 & 5 & 2 & 2 & 1 & - & 2 & - & - & 1 & - & - & - & - & - \\
\hline Zanthoxylum limoncello & - & 1 & - & - & - & - & - & 1 & 1 & - & 2 & - & - & - & - & - & - & - \\
\hline Cecropia sciadophylla & - & - & 1 & - & - & - & - & - & 2 & - & + & - & - & - & - & - & - & - \\
\hline Cochlospermum & 2 & - & - & - & - & - & - & 1 & - & - & - & 5 & - & - & - & - & - & - \\
\hline Alseis eggersii & - & - & 1 & - & + & 1 & 4 & - & - & - & - & - & - & - & + & - & - & - \\
\hline Guazuma ulmifolia & 2 & 4 & - & - & - & - & - & - & 2 & - & 5 & - & - & - & - & 1 & - & - \\
\hline \multicolumn{19}{|l|}{ Características BSI } \\
\hline Xylosma benthamii & 1 & - & - & - & - & - & - & - & - & + & 1 & 3 & 4 & 3 & 1 & 1 & 3 & 1 \\
\hline Haematoxylum campe & 1 & - & - & - & - & - & - & - & 3 & - & - & - & 1 & 1 & 1 & - & 2 & 5 \\
\hline Cissus erosa & 1 & - & - & - & - & - & - & - & 1 & 1 & - & - & 1 & - & 1 & + & - & - \\
\hline Genipa americana & + & - & - & - & - & - & - & - & - & - & - & - & 1 & 1 & 1 & - & 2 & 1 \\
\hline Turnera hindsiana & - & - & - & - & - & - & - & - & - & 1 & - & 1 & 1 & 1 & - & 2 & 1 & - \\
\hline \multicolumn{19}{|l|}{ Compañeras } \\
\hline Colicodendron scabridu & - & - & - & - & - & - & - & 2 & - & + & - & - & - & - & - & 1 & - & - \\
\hline Lantana sprucei & - & - & - & 2 & - & 2 & 4 & - & - & - & - & - & - & - & - & - & - & - \\
\hline Panicum maximum & - & - & - & - & - & - & - & - & 2 & - & - & 1 & - & + & - & - & - & - \\
\hline Inga jaunechensis & - & - & - & - & - & - & - & 2 & - & 1 & - & - & - & - & - & - & - & - \\
\hline Achyranthes aspera & - & - & - & - & - & - & - & 1 & - & - & - & 1 & - & - & - & - & - & - \\
\hline Ambrosia peruviana & - & - & - & - & - & - & - & - & - & - & - & - & 1 & 2 & - & - & - & - \\
\hline Arctium lappa & - & - & - & - & - & - & - & 2 & - & - & - & - & - & - & 1 & - & - & - \\
\hline Baccharis genistelloides & - & - & - & - & - & - & - & - & - & - & 1 & - & - & - & 1 & - & - & - \\
\hline Bambus arundo & - & - & - & - & - & - & - & - & - & - & - & - & - & - & 1 & 1 & - & - \\
\hline Ginerium sagittatum & - & - & - & - & - & - & - & 1 & - & - & - & - & - & - & 1 & - & - & - \\
\hline Crescentia cujete & - & 1 & 1 & - & - & - & - & - & - & - & - & - & - & - & - & - & - & - \\
\hline Eugenia pustulescens & - & - & 1 & - & - & - & - & - & - & - & - & - & - & - & 3 & - & - & - \\
\hline Ipomoea aquatica & - & - & - & - & - & - & - & - & - & 1 & - & - & - & - & - & 1 & - & - \\
\hline Matricaria chamomilla & - & - & - & - & - & - & - & - & - & - & - & - & 1 & 1 & - & - & - & - \\
\hline Mentha spicata & - & - & - & - & - & - & - & - & - & - & - & - & 3 & 1 & - & - & - & - \\
\hline Merremia umbellata & - & - & - & 1 & - & - & - & - & - & - & - & - & - & - & - & - & - & 1 \\
\hline Piper tuberculatum & - & - & - & - & - & - & - & 1 & - & - & - & - & - & - & - & 1 & - & - \\
\hline Prosopis juliflora & 1 & - & - & - & - & - & - & 1 & - & - & - & - & - & - & - & - & - & - \\
\hline Salvia coccinea & - & - & - & - & - & - & - & - & - & - & 1 & 1 & - & - & - & - & - & - \\
\hline \multicolumn{19}{|c|}{ 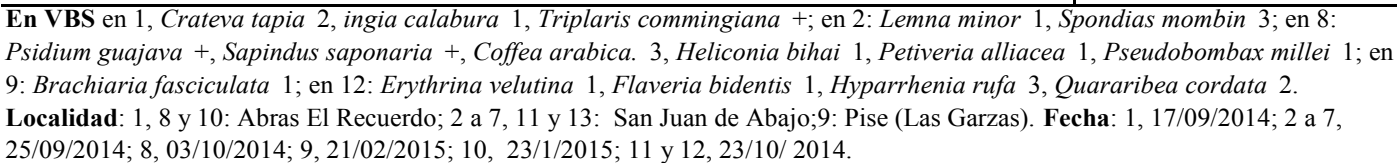 } \\
\hline
\end{tabular}

En VBSI en 1, Ischaemum rugosum 1, Luffa operculata 1; en 2: Digitaria sanguinalis,+ Echinochloa colona + , Eclipta postrata +; en 3: Ludwigia decurrens 1, Paspalum vaginatum 1, Pavonia burchellii 1, Hyptis capitata 3; Baccharis latifolia +. Localidad: 1 a 4, El Recuerdo; 5, 6: Pise Las Garzas. Fecha: 1, 2: 03/10/2014; 3: 16/01/2015; 4: 23/10/2015; 5: 14/02/2015; 6:21/02/2015. 
inundación parcial o total, según su tamaño, y de acuerdo al ritmo periódico y estacional de las lluvias. Son especies características: Xylosma benthamii, con inundación total durante la época invernal, Hematoxylum campechanum, Genipa americana. Entre los nanofanerófitos Turnera hindsiana, y Cissus erosa (Cuadro 5; asociación 5B).

A diferencia de otros humedales este hecho constituye, desde el punto de vista geobotánico, la característica singular del humedal de Abras. El proceso natural de colmatación de cualquier cubeta se inicia siempre, y en sentido centrípeto, de la orla externa de vegetación. Esta, por lo general, y sobre todo en humedales litorales (Boira, 2013) y alpinos de pendientes suaves (Azzella et al., 2014), parte de una vegetación helofítica arraigada formada por carrizos (Phragmites sp.), juncos y totoras (Typha sp., Schoenoplectum sp.) que invade progresivamente e inicia así la serie de vegetación edafohigrófila conducente al bosque de ribera.

En el caso de Abras, parte del bosque ha conseguido su presencia de forma prematura mediante la adaptación de algunos de sus miembros a las condiciones anfibias impuestas por el medio.

\section{Biodiversidad}

Los datos obtenidos para la biodiversidad florística de las distintas comunidades estudiadas refleja la baja calidad debido a la escasa presencia de especies prístinas de las formaciones acuáticas en general (Cuadro 6).

Los índices más elevados de los valores de biodiversidad (Ib) corresponden a la franja externa de esteros y canales con aguas lénticas (VBSI). Este valor $(\mathrm{Ib}=2.22)$ se ha de considerar como biodiversidad real pero no natural; el cálculo incluye tanto inventarios con plantas propias del BSI, como de la flora arvense de cultivos de arroz y maíz que ocupan la misma posición topográfica durante la época de verano. En condiciones naturales, la ausencia de elementos arvenses provocaría un notable descenso del $\mathrm{Ib}$. Los valores más elevados del Índice de homogeneidad (Ih) se registran en las muestras relícticas de vegetación acuática y palustre (Cuadro 6).

Cuadro 6. Valores medios (Err. Est.) de los Índices de Biodiversidad florística (Ib) y homogeneidad (Ih) (Shannon-Weaver) de las cinco comunidades vegetales del Humedal AdM. VAPP, acuática palustre primaria; VAG, acuática general; VAPF, de praderas flotantes; VBSI, bosque seco inundable; VBS, bosque seco

\begin{tabular}{|c|c|c|c|c|c|}
\hline \multirow[t]{2}{*}{ Comunidades vegetales } & \multicolumn{5}{|c|}{$\begin{array}{l}\text { Índices de Biodiversidad Índices de homogeneidad } \\
\text { florística (Ib) }\end{array}$} \\
\hline & $\mathrm{N}$ & media & Err. Est. & media & Err. Est. \\
\hline VAPP & 4 & 1.98 & 0.56 & 0.94 & 0.05 \\
\hline VGA & 28 & 1.41 & 0.49 & 0.85 & 0.1 \\
\hline VAPF & 4 & 1.69 & 0.41 & 0.83 & 0.04 \\
\hline VBSI & 6 & 2.22 & 0.36 & 0.92 & 0.04 \\
\hline VBS & 12 & 1.36 & 0.47 & 0.86 & 0.13 \\
\hline
\end{tabular}

\section{Anexo 1. Catálogo florístico}

Alismataceae

Albidella nymphaeifolia (Griseb.) Pichon (cucharero)

Sagittaria lancifolia L.

Sagittaria sagittifolia L.

Amaranthaceae

Achyranthes aspera L. (picha de gato, cadillo)

Anacardiaceae

Spondias mombin L. (hobo, jobo)

Araceae

Pistia stratiotes L. (lechuga de agua)

Araliaceae

Hydrocotyle ranunculoides L.

Arecaceae

Bactris gasipaes Kunth (chontilla)
Asteracae

Tridax procumbens (L.) L. (hierba de sapo)

Arctium lappa L. (morito)

Ambrosia peruviana Willd. (altamisa)

Baccharis genistelloides (Lam.) Pers.

Baccharis latifolia (Ruiz \& Pav.) Pers. (chilca)

Eclipta postrata (L.) L.

Matricaria chamomilla L. (hierba de ají)

Tridax procumbens (L.) L.

Arctium lappa L.

Ambrosia peruviana Willd.

Baccharis genistelloides (Lam.) Pers.

Baccharis latifolia (Ruiz \& Pav.) Pers.

Eclipta postrata (L.) L.

Matricaria chamomilla L. 
Begoniaceae

Begonia cucullata Willd.

Crescentia cujete L. (bototo, mate)

Bignoniaceae

Roseodendron donnell-smithii (Rose) Miranda. (guayacán blanco)

Bombacaceae Quararibea cordata (Bonp.) Vischer

Bixaceae

Cochlospermum vitifolium (Willd.) Spreng. (bototillo)

Cabombaceae Cabomba aquatica Aubl.

Capparaceae

Crateva tapia L. (jagua de lagarto)

Colicodendron scabridum (Kunth) Seem. (níspero, zapote de perro)

Convolvulaceae

Ipomoea aquatica Forssk.

Ipomoea carnea Jacq.

Ipomoea tiliacea (Willd.) Choisy (betilla)

Merremia umbellata (L.) Hallier

Cucurbitaceae

Luffa operculata (L.) Cogn. (estropajo)

Cuscutaceae

Cuscuta sp.

Cyperaceae

Cyperus aggregatus (Willd.) Endl. (paja de burro)

Cyperus compactus Retz.

Cyperus diffusus Vahl

Cyperus papyrus L. (papiro

Eleocharis elegans (Kunth) Roem \& Schult (Velita)

Euphorbiacea

Croton hirtus L' Her

Flacourtiaceae

Muntingia calabura L. (niguito)

Heliconiaceae

Heliconia bihai L. (platanillo)

Lamiaceae

Hyptis capitata Jacqs.

Mentha spicata L. (mastrante)

Salvia coccinea Buchoz ex Etl.

Tectona grandis L. (teca)

Lecythidiaceae

Gustavia pubescens Ruiz \& Pav. ex O.Berg. (membrillo)

Leguminosae

Erythrina velutina Willd.

Haematoxylum campechianum L. (tinto)

Inga semialata (Vell.) C.Mart. (guabo de río)

Lonchocarpus atropurpureus Benth.

Machaerium isadelphum (E. Mey.) Stande

Machaerium millei Standl. (palo de hacha)

Mimosa pigra L. (uña de gato)

Neptunia oleraceae Lour. (mimosa de agua)

Prosopis juliflora (Sw.) DC. (algarrobo)
Lemnaceae

Lemna minor $\mathrm{L}$.

Malvaceae

Urena lobata L. (cadillo)

Guazuma ulmifolia Lam. (guazimo, guasmo)

Malachra alceifolia Jacq.

Pavonia burchellii (DC) R.A.Dier. (malva)

Pseudobombax millei (Standl.) A. Robyns, (beldaco)

Mimosaceae

Inga jaunechensis A.H.Gentry. (guabo de mico)

Myrtaceae

Psidium guajava L., (guayabo de puerco)

Eugenia pustulescens McVaugh

Nymphaceae

Nymphaea glandulifera Rodschied.

Onagraceae

Ludwigia helminthorrhiza (Mart.) H.Hara

Oenothera villosa Thunb.

Ludwigia decurrens Walter. (clavo)

Passifloraceae

Passiflora vitifolia Kunth, (maracuyá)

Phyllanthaceae

Phyllantus juglandifolius Willd, (culo pesado)

Phytolaccaceae

Petiveria alliacea L., (zorrilla)

Piperaceae

Piper marginatum J. (cordoncillo)

Poaceae

Bambus arundo C.C. Gmel. (Arundo donax)

Brachiaria fasciculata (Sw.) Parodi (gramalote)

Ginerium sagittatum (Aubl.) P. Beauv.

Digitaria sanguinalis (L.) Scop. (paja de burro)

Echinochloa colona (L.) Link

Echinochloa crus-galli (L.) P. Beauv.

Flaveria bidentis (L.) Kuntze

Guadua angustifolia Kunth (guadua)

Hyparrhenia rufa (Nees) Stapf

Ischaemum rugosum Salisb. (mazorquilla de agua)

Panicum maximum Jacq. (sequejo)

Panicum mertensii Rooth

Paspalum distichum L. (gramalote)

Paspalum repens P.J.Bergius

Paspalum vaginatum Sw. (pasto)

Pennisetum purpureum Schumach. (pasto elefante)

Typha domingensis Pers. (totora, anea)

Polygonaceae

Polygonum aquaticum A.Gray ex Meisn

Triplaris commingiana Fish. \& C.A. Mey. (fernansánchez)

Pontederiaceae

Eichhornia azurea (Sw.) Kunth (lirio de agua)

Eichhornia crassipes (Mart.) Solms (lechuguin)

Rubiaceae

Alseis eggersii Standl. (palo de vaca) 
Coffea arabica L. (café)

Genipa americana L. (jagua)

Zanthoxylum limoncello Planch. \& Oerst. ex Triana \& Planch (limoncillo)

Salicaceae

Xylosma benthamii (Tul.) Triana \& Planch. (rompejato)

Salviniaceae

Azolla caroliniana Willd.

Salvinia hispida Kunth

Sapindaceae

Cupania cinerea Poepp. (come pava)

Sapindus saponaria L. (jaboncillo)

Solanaceae

Solanum flavescens Dunal

Sphenocleaceae

Sphenoclea ceylanica Gaertn.

Turneraceae

Turnera hindsiana Beth. (palo de murciélago)

Urticaceae

Cecropia sciadophylla Mart. (guarumo)

Verbenaceae

Lantana sprucei Hayek (mastrante)

Vitaceae

Cissus erosa Rich (barba de iguana)

\section{Agradecimiento}

$\mathrm{E}_{\mathrm{p}}^{1}$ presente trabajo fue patrocinado por el Proyecto Prometeo de la Secretaría de Educación Superior, Ciencia, Tecnología e Innovación de la República del Ecuador.

\section{Bibliografía}

Aguirre, Z., Linares-Palomino, R. y Kvist, L. 2006. Especies leñosas y formaciones vegetales en los bosques estacionalmente secos de Ecuador y Perú. Arnoldo a 13 (2): $324-350$.

Alvarez, M. and Mynett, A. 2010. Hydroinformatics and Knowledge Management Department. Abras de Mantequilla (IHE, Deltares) Delft. $7^{\mathrm{a}}$ International Conference on Ecological Informatics. Ghent (Bélgica)

Arias, M.; Villa, G. and Alvarez, G. 2010. WET Ecoservices evaluation in the AdM wetland.In R. Johnston, J. Cools, S. Liersch, S. Morardet, C. Murgue, et al.. WETwin: a structured approach to evaluating wetland management options in data-poor contexts. Environmental Science \& Policy, 2013, 34, p. 3 - p. 7. 1.

Azzella, M., Rosati, L., Iberite, M., Bolpagni, R. and Blasi, C. 2014. Changes in aquatic plants in the Italian volcaniclake system detected using current data and historical records. Aquatic Botany 112, pp. 41-47.

Balls, H., Moss, B. and Irvine, K. 1985. The effects of high nutrient loading on interactions between aquatic plante and phytoplankton.Verh. Internat. Verein. Limnol. 22: 1912-2915.

Boira, H. 2013. La vegetación de los humedales litorales valencianos. Edc. Universitat Politecnica de Valencia. ISBN, 978-84-8363-895-8.

Bourliere, F. 1983. Ecosystems of the World 13.Tropical Savannas. Amsterdam: Elsevier.

Braun-Blanquet, J. 1979. Fitosociología. Bases para el estudio de las comunidades vegetales [ed. 3]. H. Blume Ediciones, Barcelona.

Brown, G. 2008. Systematics of the tribe Ingeae (Leguminosae, mimosoideae) over the past 25 years. Muelleria 26(1): 27-42.

Cariguan, R. and Kalff, J. 1980. Phosphorus sources for aquatic weeds: water or sedimente. Science 207: 987989.

Child, M., Milron, S., Dean. R., Lipsey, M., Puttick, J., Hempson, T., Mann, G., Babiker, H., Chaudrey, J., Humphrey, G., Joseph, G., Okes, N., Potts, R. and Wistebaar, T. 2010. Tree-grass coexistence in a flooddisturbed, semi-arid savanna system. Landscape Ecology 25: 315-326.

Dodson, C., Gentry, A. y Valverde, F. 2005. Flora de Jauneche, Los Ríos, Ecuador. Universidad de Guayaquil. Ecuador.

Eiten, G. 1972. The cerrado vegetation of Brazil. Botanical Review 38: 201- 349.

Engel, S. 1988. The role and interactions of submersed macrophytes in a shallow Wisconsin lake. J. Freshwater Ecol. 4: 329-341.

Franco, AC. 2002. Ecophysiology of Woody Plants. In Oliveira P.S. \& Marquis R. J. (Eds.), The Cerrados of Brazil. Columbia University Press, New York, pp. 1178197.

Furley, P. 1999. The nature and diversity of neotropical savanna vegetation with particular reference to the Brazilian Cerrados. Global Ecology and Biogeography 8: 223-241.

Furley, PA. 1996. The influence of slope on the nature and distribution of soils and plant communities in the central Brazilian Cerrado. In: Anderson, M.G., Brooks, S. (Eds.), Advances in Hill slope processes. Wiley, London, pp. 327- 346.

Gottsberger, G. and Silberbauer-Gotrsberger, I. 2006. Life in the Cerrado: A South America Tropical Seasonal Ecosystem. V. 1. Origin, Structure, Dynamics and Plant Use. Reta Verlag, Ulm.

Haridasan, M. 2008. Nutritional adaptations of native plants of the Cerrado biome in acid soils. Brazilian Journal of Plant Physiology 20, 183-195.

Harling, G. and Persson, C. 1973. Flora de Ecuador. Department of Plant and Environmental Sciences, Göteborg University. Suecia.

Harling, G. and Persson, C. 2005. Flora de Ecuador. 
Department of Plant and Environmental Sciences, Göteborg University. Suecia

Hill, M. 1979. TWINSPAN - A FORTRAN program for arranging multivariate data in an ordered two way table by classification of individuals and attributes. Cornell University Ithaca. NY.

Jones, R. 1990. The Effect of Submersed Aquatlc Vegetation on Phytoplankton and Water Quality in theTidal Freshwater Potomac River. Journal of Freshwater Ecology, 5(3): 279-288).

Kovacs Compueter. 2012. MVSP (Multi-Variate Statistical Package) v.3.21.

MAE (Ministerio del Ambiente del Ecuador). 2012. Sistema de clasificación de los ecosistemas del Ecuador continental. Subsecretaría de Patrimonio Natural. Pg. 143. Quito-Ecuador

Moreno-Casasola, P., Cejudo-Espinosa, E., CapistránBarradas, A., Infante-Mata, D., López-Rosas, H., Castillo-Campos, G., Pale-Paley, J. y CamposCascaredo, A. 2010. Composición florística, diversidad y ecología de humedales herbáceos emergentes en la planicie costera central de Veracruz, México. Bol. Soc. Bot. Méx 87: 29-50.

Nõges, P., Nõges, T., Tuvikene, L., Smal, H., Ligeza, S., Kornijów, R., Peczula, W., Bécares, E., Garcia-Criado, F., Alvarez-Carrera, C., Jensen, J., Gross, E., LarsAnders, H., Gyllström, M., Nykänen, M., de Eyto, E., Irvine K., Stephen, D., Collings, S. and Moss, B. 2003. Factors controlling hydrochemical and trophic state variables in 86 shallow lakes in Europe. Hydrobiologia 506-509 (1-3): 51-58.

Pétémanagnan, J-M., Coulibaly, L., Tiho, S., Ouattara, A., Gouréne A. and Gourène, G. 2011. Panicum maximum (Jacq.) density effect upon macrofauna structure in sediments of pilot-scale vertical flow constructed wetlands treating domestic wastewater. Ecological Engineering, 37(2): 217-223.
Rivas, S. 2008. Worldwide Bioclimatic Classification System. Phytosociological Research Center, Spain.

Romo, S. and Miracle, R. 1994. Long-term phytoplankton changes in a shallow hypertrophic lake, Albufera of Valencia (Spain). Hydrobiologia; 275-276(1): 153-164.

Rorslett, B., Berge, D. and Johansen, SW. 1986. Lake enrichment by submersed macrophytes: a Norwegian whole-lake experience with Elodea canadensis. Aquatic Botany 26: 325-340.

Rossatto, DR., Silva, Lucas de Carvalho, Villalobos-Vega, R., da Silveira, Lobo Sternbergc and Francod, A. 2012. Depth of water uptake in woody plants relates to groundwater level and vegetation structure along a topographic gradient in a neotropical savanna. Envíronment.al and Experimental Botany 77: 259-266

Ruggiero, PGC., Pivello, VR., Sparovek, G., Teramoto, E. and Pires Neto, AG. 2006. Relação entre solo, vegetação e topografia em área de cerrado (Parque Estadual de Vassununga, SP): como se expressa em mapeamentos. Acta Botanica Brasílica 20, 383-394.

Shannon C. and Weaver, W. 1971. The mathematical theory of communication.University of Illinois Press. Cited in Magurran, AE., 2004, Measuring biological diversity, Blackwell Publishing: Oxford, UK.

Soriano, AM. 2010. Flora de Veracruz (México) POACEAE IV: PANICEAE, Panicum L. 152, 1- 91. Instituto de Ecología A. C.

Timms, RM. and Moss, B. 1984. Prevention of growth of potentially dense phytoplankton populations by zooplankton grazing, in the presence of zooplanktivorous, in a shallow wetland ecosystem.Limnol. Oceanogr. 29: $472-486$. 